\title{
DESIGN OF 3 GEV BOOSTER FOR NSLS-II
}

\author{
T. Shaftan\#, J. Rose, I. Pinayev, X.J. Wang, J.B. Murphy, E. D. Johnson, BNL, Upton, NY 11973, \\ U.S.A.
}

\section{Abstract}

We present preliminary design of full-energy booster for NSLS-II. In the paper we analyse single- and multibunch modes of the booster operations. The booster lattice consists of 24 theoretical minimum emittance (TME) cells with two dispersion suppressors. Lattice design, initial design of the magnets and eddy current contribution to the booster chromaticity is briefly discussed.

\section{INTRODUCTION}

A fuller description of the NSLS-II can be found in our other publications $[1,2,3]$. The project features a thirdgeneration storage ring with optics based on 24 TBA cells. Table 1 lists the NSLS-II parameters relevant to the injection.

The goal parameters of the NSLS-2 lead to developing a flexible and highly efficient injection system with high repetition rate. In [4] we discuss two options for the fullenergy injector: a booster (repetition rate of a few $\mathrm{Hz}$ depending on the choice of a ramping power supply), and a linac (tens or hundreds of $\mathrm{Hz}$ ). In this paper we discuss the booster design as an option for the NSLS-II injector.

User requirements constrain frequency of injection cycles in the top-off mode [4] to be less than 1 cycle per minute. Figure 1 provides a sketch of two possible modes of booster operations during top-off: single- and multibunch injection and acceleration.

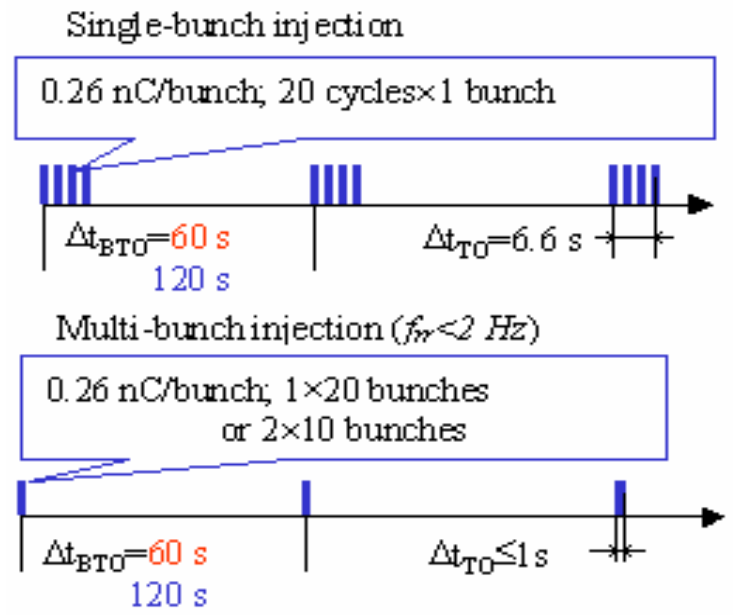

Fig. 1: Modes of booster operation for the storage ring top-off. $\Delta t_{B T O}$ is time interval between top-off cycles (one minute without- and two minutes with harmonic cavity), $\Delta t_{T O}$ is time for a single top-off cycle. Charge per the injected bunch is $0.26 \mathrm{nC}$.

\#shaftan@bnl.gov
Table 1: Design Parameters of NSLS-II

\begin{tabular}{|l|l|}
\hline Energy & $3 \mathrm{GeV}$ \\
\hline Operating current & $500 \mathrm{~mA}$ \\
\hline Circumference & $\sim 650 \mathrm{~m}$ \\
\hline RF frequency & $500 \mathrm{MHz}$ \\
\hline Number of bunches & $\begin{array}{l}\sim 700 \quad \text { out of } \sim 1,000 \\
\text { buckets }\end{array}$ \\
\hline Charge per bunch & $1.55 \mathrm{nC}$ \\
\hline Estimated lifetime & $\begin{array}{l}3 \text { hours }(\sim 6 \text { hours with } \\
3^{\text {rd }} \text { harmonic cavity }\end{array}$ \\
\hline Length of straight section & $7 \mathrm{~m}$ \\
\hline
\end{tabular}

Table 2 summarizes the main parameters of a current booster design. The booster repetition rate of $3 \mathrm{~Hz}$ provides the necessary rate of the initial fill in the multibunch mode.

Table 2: Full-Energy Booster Parameters

\begin{tabular}{|l|l|}
\hline Injection energy & $0.1 \mathrm{GeV}$ \\
\hline Circumference & $170 \mathrm{~m}$ \\
\hline Repetition rate & $3 \mathrm{~Hz}$ \\
\hline Horizontal emittance (at $3 \mathrm{GeV})$ & $10.5 \mathrm{~nm}$ \\
\hline Energy spread (at $3 \mathrm{GeV})$ & $0.1 \%$ \\
\hline RF voltage & $1.5 \mathrm{MV}$ \\
\hline RF acceptance & $1 \%$ \\
\hline Length of straight section & $8 \mathrm{~m}$ \\
\hline Betatron tunes, $\mathrm{x} / \mathrm{y}$ & $12.16 / 4.36$ \\
\hline Natural chromaticity, $\mathrm{x} / \mathrm{y}$ & $-28.6 /-8.6$ \\
\hline Momentum compaction & 0.035 \\
\hline
\end{tabular}

In the single-bunch mode injection cycle takes 6.7 seconds [4], which means that $11 \%$ of overall beam time is spent on injection. This value is unacceptably large; multi-bunch mode allows reducing the cycle duration down to less than a second. This corresponds to injecting 20 bunches into the ring once a minute (in one booster cycle), or two consecutive booster cycles with 10 bunches each.

We are also discussing another mode of the booster operation when the macropulse is injected into the booster and, after acceleration, short-pulse kicker extracts single bunch out of the macropulse for subsequent injection into the storage ring bucket to be refilled. This mode of operation requires storing the macropulse from linac in the booster during flat portion of the energy ramp at 3 $\mathrm{GeV}$. This mode of the booster operation provides with possibility of injection in arbitrary (not necessarily consecutive) ring buckets during a single top-off cycle.

\section{BOOSTER MODEL}

To ensure a small footprint and find a cost-effective solution, we designed the TME booster lattice out of 24 cells with gradient dipoles and two straight sections for injection and extraction. Figures 2 and 3 show the booster 
and the dispersion suppressor lattices calculated and optimised using the MAD program [5].

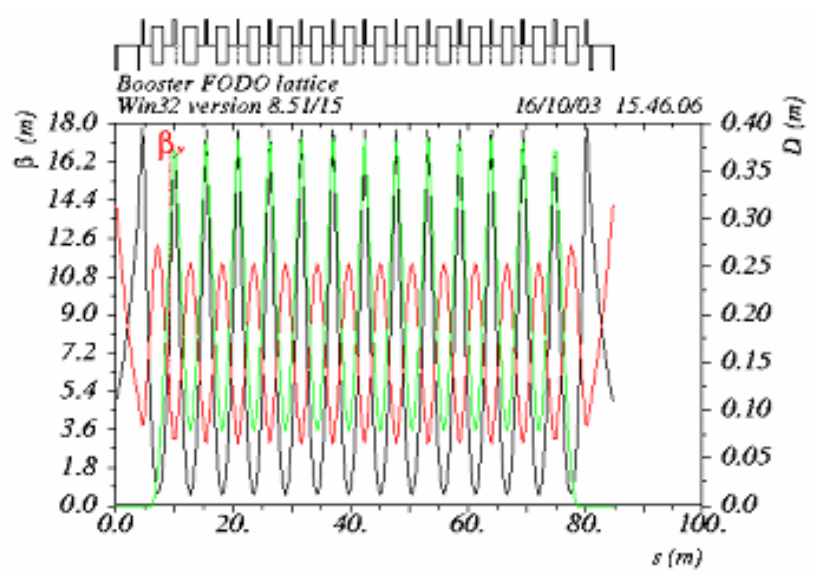

Fig. 2: Half of the booster lattice.

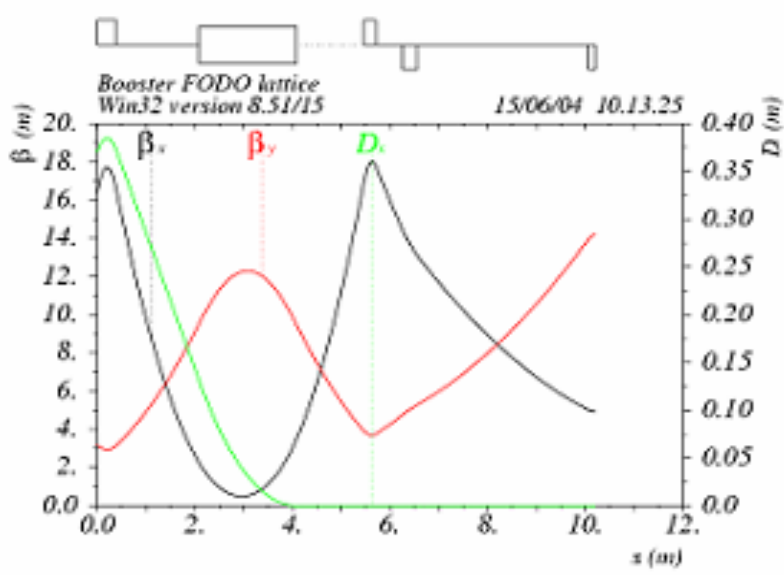

Fig. 3: Booster dispersion suppressor.

For an estimate of the required chromaticity correction we introduced thin sextupoles in the TME cell. Preliminary analysis of the booster dynamic aperture using the OPA program [6] exhibits a value of more than $25 \mathrm{~mm}$ in both transverse dimensions.

Figure 4 shows preliminary magnetic design of the booster bend using the MERMAID code [7]. Calculated dipole and quadrupole components correspond to the design values.

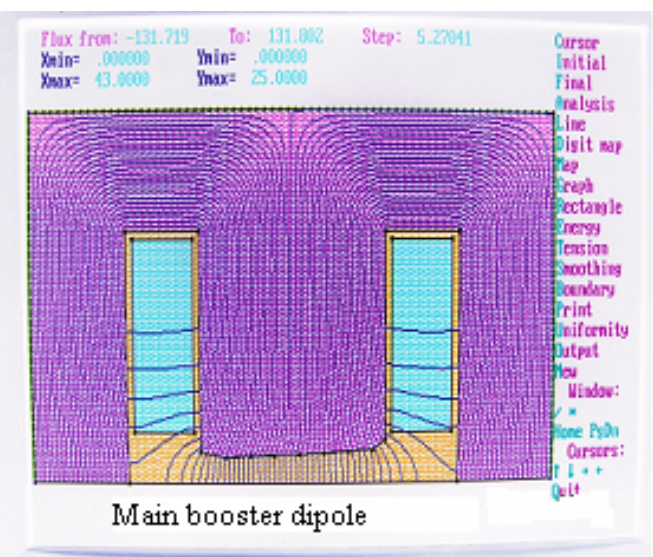

Fig. 4: Model of the booster dipole.
The booster RF can be realized by using a 7-cell PETRA-type RF cavity operating at $500 \mathrm{MHz}$. The booster model provides with $1 \%$ energy acceptance at extraction energy $3 \mathrm{GeV}$ for RF voltage of $1.5 \mathrm{MV}$.

Given the repetition rate of $3 \mathrm{~Hz}$ (Table 2), we estimated the sextupole moment contribution due to Eddy currents along the energy ramp $(160 \mathrm{msec})$. Assuming sinusoidal energy ramp and realistic design of vacuum chamber we estimated sextupolar component (upper plot in Fig. 5) and chromaticity contribution (lower plot in Fig. 5). In our calculations we used a method presented in [8].
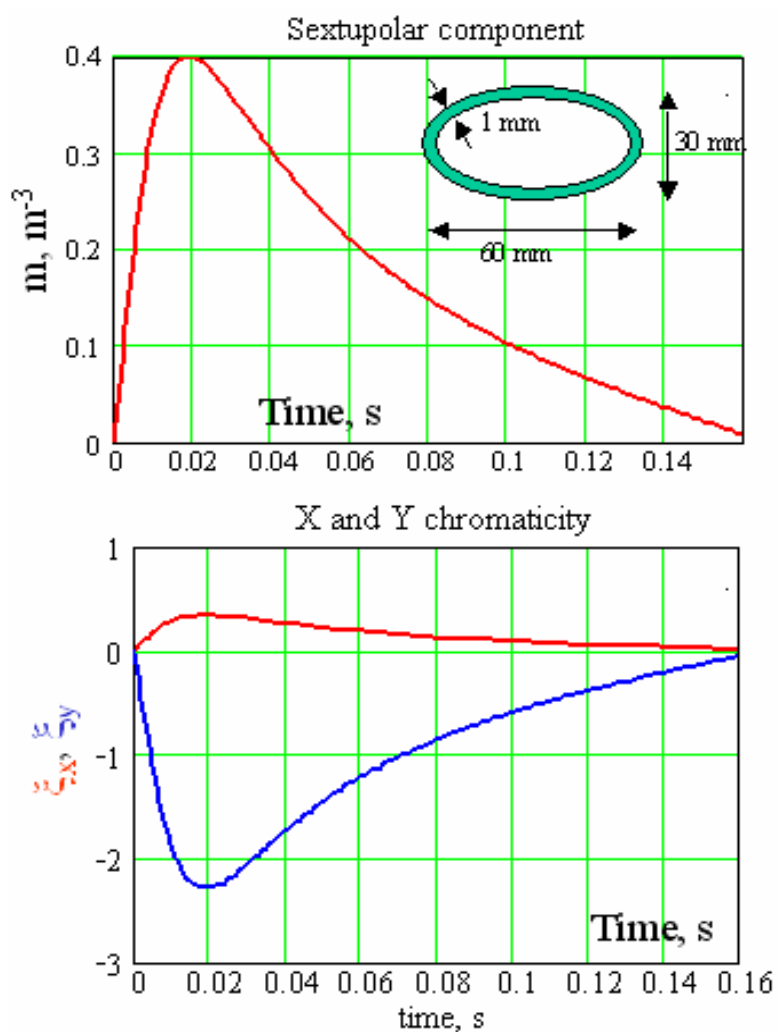

Fig. 5: Estimate of eddy currents effect. Upper plot shows dependence of the induced sextupole moment along the energy rump (stainless steel vacuum chamber geometry is sketched above the curve). Lower plot shows eddy currents-induced contribution to the booster chromaticity.

Calculated value of chromaticity does not look too large, but provision for additional families of sextupoles is necessary.

\section{CONCLUSION}

We developed a full-energy booster model, which can be used as an injector into the NSLS-II storage ring. During the initial design we have estimated various aspects of the booster, such as, its lattice, magnetic design of the gradient dipoles, and the effects of Eddy currents. The chosen booster lattice, based on gradient TME cells, is feasible for creating a compact low-emittance injector. 


\section{NOTICE}

The manuscript has been authored by Brookhaven Science Associates, LLC under Contract No. DE-AC0298CH1-886 with the U.S. Department of Energy. The United States Government retains, and the publisher, by accepting the article for publication, acknowledges, a world-wide license to publish or reproduce the published form of this manuscript, or allow others to do so, for the United States Government purposes.

\section{REFERENCES}

[1] B. Podobedov, J.M. Ablett, L. Berman et al., Proc. of PAC-2003, Portland, Oregon, p. 241.
[2] J.B. Murphy, J. Bengtsson, L. Berman, et al., Proc. of EPAC-2004, Lucerne, Switzerland, p. 2457.

[3] J.B. Murphy, J. Bengtsson, R. Biscardi et al., these Proceedings.

[4] T. Shaftan, I. Pinayev, J. Rose et al., these Proceedings.

[5] http://project-mad9.web.cern.ch/project-mad9/

[6] http://slsbd.psi.ch/ streun/opa/opa.html

[7] Mermaid, the 2D/3D code for magnetic design, (C)A.N.Dubrovin, Novosibirsk, Russia.

[8] M. Muños and W. Joho, SLS-TME-TA-1998-0010, PSI Preprint, 1998. 